\title{
A characterization of supersmoothness of multivariate splines
}

\section{Michael S. Floater ${ }^{1} \cdot \mathrm{Kaibo} \mathrm{Hu}^{2}$}

Received: 2 July 2019 / Accepted: 6 August 2020 /

Published online: 26 August 2020

(C) The Author(s) 2020

\begin{abstract}
We consider spline functions over simplicial meshes in $\mathbb{R}^{n}$. We assume that the spline pieces join together with some finite order of smoothness but the pieces themselves are infinitely smooth. Such splines can have extra orders of smoothness at a vertex, a property known as supersmoothness, which plays a role in the construction of multivariate splines and in the finite element method. In this paper, we characterize supersmoothness in terms of the degeneracy of spaces of polynomial splines over the cell of simplices sharing the vertex, and use it to determine the maximal order of supersmoothness of various cell configurations.
\end{abstract}

Keywords Supersmoothness $\cdot$ Spline $\cdot$ Finite element $\cdot$ Macroelement

Mathematics Subject Classification (2010) Primary: 41A15 - 65D07 ·

Secondary: 41A58 $\cdot 65 \mathrm{~N} 30$

\section{Introduction}

Polynomial splines over a simplicial partition of a domain in $\mathbb{R}^{n}$ (a triangular mesh in $2 \mathrm{D}$, a tetrahedral mesh in $3 \mathrm{D}$, and so on) are functions whose pieces are polynomials

Communicated by:Larry L. Schumaker

KH was supported in part by the European Research Council under the European Union's Seventh Framework Programme (FP7/2007-2013)/ERC grant agreement 339643, during his affiliation with the University of Oslo.

Michael S. Floater

michaelf@math.uio.no

Kaibo $\mathrm{Hu}$

khu@umn.edu

1 Department of Mathematics, University of Oslo, Moltke Moes vei 35, 0851, Oslo, Norway

2 School of Mathematics, University of Minnesota, 206 Church St. SE, Minneapolis, MN, 55455-0488, USA 
up to a certain degree $d$ and which join together with some order of continuity $r$. Such spline functions may have extra orders of smoothness at a vertex of the mesh, a property known as supersmoothness as suggested by Sorokina [14]. For example, the Clough-Tocher macroelement, which is $C^{1}$ piecewise cubic, is twice differentiable at the refinement point, as first observed by Farin [5], and so this element can be said to have supersmoothness of order 2 at that point.

For the construction of splines or finite elements with higher orders of continuity, it is important to recognize and make use of supersmoothness. For example, it plays a role in many of the macroelement constructions surveyed by Lai and Schumaker [8], where applications of splines to approximation theory and computer-aided geometric design are discussed. The concept of supersmoothness is also relevant to the finite element method. Motivated by structure-preserving or compatible discretizations, there has recently been an increased interest in investigating the use of splines for vector fields and differential complexes [2-4, 7]. The de Rham complex reveals a connection between smooth, e.g., $C^{1}$, finite elements and the Stokes problem in fluid mechanics. In a discrete de Rham complex, the spline spaces for the velocity field may inherit the supersmoothness of the scalar field $[2,4,7,11]$. Thus, supersmoothness is also of importance in the study of these problems.

Since Farin's observation about the Clough-Tocher element, Sorokina, in [14] and [15], has derived further supersmoothness properties of polynomial splines, and in particular higher order supersmoothness in a cell in 2D; see equation (4). More recently, Shekhtman and Sorokina [12] observed that supersmoothness is a phenomenon of more general spline functions, not only piecewise polynomials. Their results imply that at the vertex $v$ of a triangulation with $m$ incoming edges all having different slopes, any $C^{r}$ spline with $r \geq m-2$ has derivatives of order $r+1$ at $v$ as long as the spline pieces themselves have $C^{r+1}$ continuity in a neighborhood of $v$.

The results of [12] were the motivation for this paper. If we simplify the framework of [12] and assume that all the spline pieces are $C^{\infty}$ smooth, which is the case for polynomials and many other functions of interest, can we extend the results to higher orders of supersmoothness and also to higher Euclidean space dimensions? Our solution is to simplify the problem by deriving a characterization of supersmoothness in terms of the degeneracy of polynomial spline spaces over the cell (in Theorem 1). Using this, the maximal order of supersmoothness at a vertex can be determined once a general formula for the dimensions of the polynomial spline spaces over the cell is known. At the end of the paper, we apply these results to various cell configurations.

\section{Cells and supersmoothness}

We start with some definitions.

\subsection{Simplicial meshes and cells}

Let $\Delta$ be a set of $n$-simplices in $\mathbb{R}^{n}$. We call $\Delta$ a mesh if the intersection between any two $n$-simplices $T_{1}, T_{2} \in \Delta$ is either empty or a common $k$-face for some $k$, $0 \leq k \leq n-1$. We let $\Omega=\cup\{T: T \in \Delta\}$. 

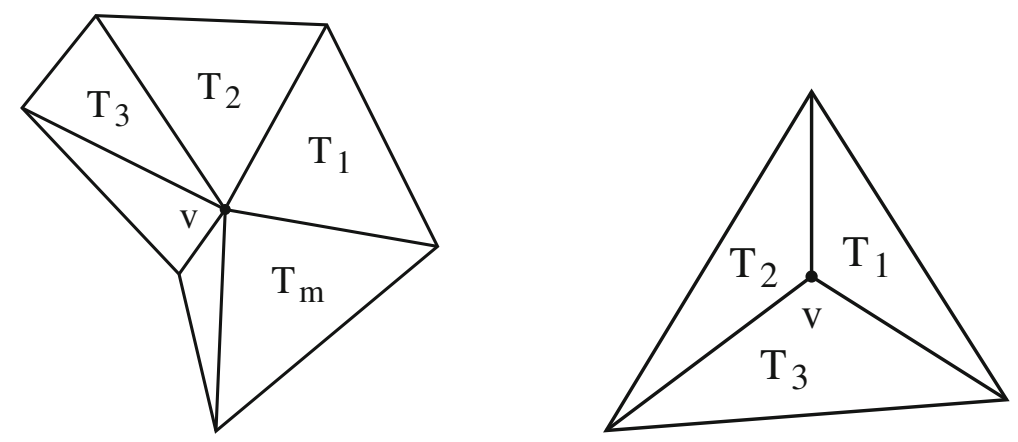

Fig. 1 Cells in 2D

If $v$ is a vertex in the mesh, we denote by $\Delta_{v} \subset \Delta$ the $n$-simplices in $\Delta$ that share $v$, and we call $\Delta_{v}$ a cell. Let $\Omega_{v}=\cup\left\{T: T \in \Delta_{v}\right\}$. We will say that $v$ is an interior vertex of $\Delta$ if $v$ is in the interior of $\Omega_{v}$, in which case we will say that $\Delta_{v}$ is an interior cell.

In $2 \mathrm{D}$, an interior cell $\Delta_{v}$ is a sequence of triangles $\Delta_{v}=\left\{T_{1}, T_{2}, \ldots, T_{m}\right\}, m \geq 3$, that form a star-shaped polygon $\Omega$, as in Fig. 1 .

In the special case that $m=3, \Delta_{v}$ is known as a Clough-Tocher split since it can also be constructed by refinement. We could start with any triangle $T$ in the plane (the outer triangle in the figure), then let $v$ be any point inside $T$ and connect the three edges of $T$ to $v$, thus creating three sub-triangles of $T$.

In $3 \mathrm{D}$, a cell is a collection of tetrahedra. A simple example of an interior cell $\Delta_{v}$ in 3D is the Alfeld split, constructed by choosing a tetrahedron $T$, then any point $v$ inside $T$ and connecting $v$ to the four triangular faces of $T$. The resulting cell has four tetrahedra, as in Fig. 2.

Fig. 2 Alfeld split in 3D

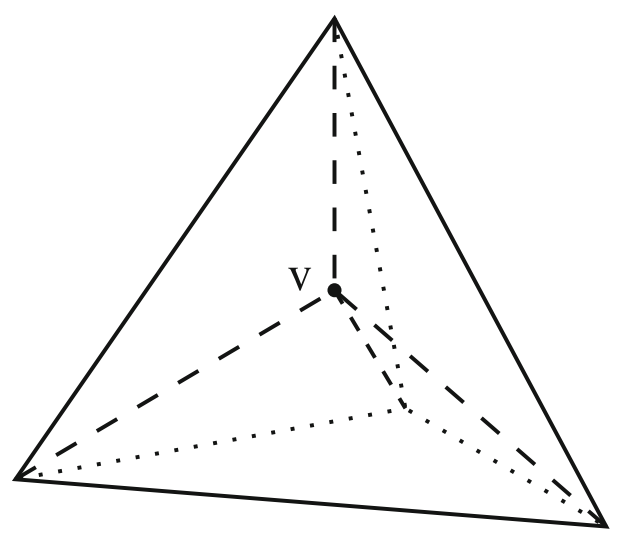




\subsection{Splines}

In this paper in order to have a notion of supersmoothness of various orders, we need to view a spline as a set of pairs of open neighborhoods and smooth functions: one pair for each $n$-simplex in the mesh $\Delta$. Thus, a spline $\sigma$ has the form:

$$
\sigma=\left\{\left(U_{T}, f_{T}\right): T \subset U_{T} \subset \mathbb{R}^{n}, U_{T} \text { open, } f_{T} \in C^{\infty}\left(U_{T}\right), T \in \Delta\right\},
$$

and we denote by $S(\Delta)$ the set of all such splines. The pieces $f_{T}$ could, for example, be polynomials of any degree (in which case we can take $U_{T}=\mathbb{R}^{n}$ ), trigonometric functions, rational functions, and so on.

Next, we consider how the pieces of $\sigma$ might fit together. Let $f \in C^{\infty}(U)$ for some open set $U \subset \mathbb{R}^{n}$ and let $x=\left(x_{1}, x_{2}, \ldots, x_{n}\right) \in U$. Let $\alpha=\left(\alpha_{1}, \ldots, \alpha_{n}\right)$ be a multi-index, with $\alpha_{1}, \ldots, \alpha_{n} \geq 0$. Then, we denote by:

$$
D^{\alpha} f(x)=\left(\frac{\partial}{\partial x_{1}}\right)^{\alpha_{1}} \cdots\left(\frac{\partial}{\partial x_{n}}\right)^{\alpha_{n}} f(x),
$$

the corresponding partial derivative of $f$ at $x$, of order $|\alpha|=\alpha_{1}+\cdots+\alpha_{n}$. We will say that a spline $\sigma \in S(\Delta)$ has smoothness $r \geq 0$ if:

$$
\left.D^{\alpha} f_{T_{1}}\right|_{F}=\left.D^{\alpha} f_{T_{2}}\right|_{F}, \quad|\alpha| \leq r, \quad T_{1}, T_{2} \in \Delta, \quad T_{1} \cap T_{2}=F, \quad F \text { is an }(n-1) \text {-face. }
$$

We will denote by $S^{r}(\Delta)$ the set of all such splines.

\subsection{Smoothness at a vertex}

Suppose that $v$ is a vertex of $\Delta$ and let $\sigma \in S\left(\Delta_{v}\right)$. We will say that $\sigma$ has smoothness of order $\rho$ at $v$ if:

$$
D^{\alpha} f_{T_{1}}(v)=D^{\alpha} f_{T_{2}}(v), \quad|\alpha| \leq \rho, \quad T_{1}, T_{2} \in \Delta_{v} .
$$

We will denote by $C^{\rho}\left(\Delta_{v}\right)$ the set of all such splines.

\subsection{Supersmoothness}

Now, we look at enhanced smoothness of splines at an interior vertex $v$ of $\Delta$. We will say that a spline $\sigma \in S^{r}\left(\Delta_{v}\right)$ has supersmoothness of order $\rho \geq r$ at $v$ if $\sigma \in C^{\rho}\left(\Delta_{v}\right)$. Thus, we are interested in the question of whether $S^{r}\left(\Delta_{v}\right) \subset C^{\rho}\left(\Delta_{v}\right)$ for some $\rho>r$. This will depend on the geometric configuration of the $n$-simplices of $\Delta_{v}$.

\section{Taylor approximations}

Our aim is to characterize supersmoothness in terms of the degeneracy of polynomial splines. The first step in the derivation is to study Taylor approximations. Let $f \in$ 
$C^{\infty}(B)$ for some domain $B \subset \mathbb{R}^{n}$. With respect to a point $v=\left(v_{1}, \ldots, v_{n}\right) \in B$, we denote the Taylor approximation of $f$ of order $\rho \geq 0$ by:

$$
\mathcal{T}_{v, \rho} f(x)=\sum_{|\alpha| \leq \rho} \frac{D^{\alpha} f(v)}{\alpha !}\left(x_{1}-v_{1}\right)^{\alpha_{1}} \cdots\left(x_{n}-v_{n}\right)^{\alpha_{n}}, \quad x \in B,
$$

where $\alpha !=\alpha_{1} ! \cdots \alpha_{n}$ !. We will make use of the following property of these Taylor approximations.

Lemma 1 Let $v, w$ be distinct points in $\mathbb{R}^{n}$ and let $e=[v, w]$ be the line segment connecting them. Let $B \subset \mathbb{R}^{n}$ be some domain containing e. Suppose that $f, g \in$ $C^{\infty}(B)$ and that $\left.f\right|_{e}=\left.g\right|_{e}$. Then, for any $\rho \geq 0,\left.\mathcal{T}_{v, \rho} f\right|_{e}=\left.\mathcal{T}_{v, \rho} g\right|_{e}$.

Proof We can represent the line segment $e$ parametrically as:

$$
e=\{v+t u: 0 \leq t \leq 1\},
$$

where $u=w-v$. Letting $x=v+t u$ for some $t \in[0,1]$, we find that:

$$
\begin{aligned}
\mathcal{T}_{v, \rho} f(x)=\mathcal{T}_{v, \rho} f(v+t u) & =\sum_{|\alpha| \leq \rho} \frac{D^{\alpha} f(v)}{\alpha !}\left(t u_{1}\right)^{\alpha_{1}} \cdots\left(t u_{n}\right)^{\alpha_{n}} \\
& =\sum_{i=0}^{\rho} \frac{t^{i}}{i !} \sum_{|\alpha|=i} \frac{i !}{\alpha !} D^{\alpha} f(v) u_{1}^{\alpha_{1}} \cdots u_{n}^{\alpha_{n}}=\sum_{i=0}^{\rho} \frac{t^{i}}{i !} h^{(i)}(0),
\end{aligned}
$$

where

$$
h(\tau)=f(v+\tau u), \quad \tau \in[0,1] .
$$

Since $f$ and $g$ are equal on $e$, we also have:

$$
h(\tau)=g(v+\tau u), \quad \tau \in[0,1],
$$

and so

$$
\mathcal{T}_{v, \rho} g(x)=\sum_{i=0}^{\rho} \frac{t^{i}}{i !} h^{(i)}(0)=\mathcal{T}_{v, \rho} f(x) .
$$

We want to generalize this property to derivatives of $f$ and $g$. To do this, we first show:

Lemma 2 Let $v \in \mathbb{R}^{n}$ and suppose $f \in C^{\infty}(B)$ for some domain $B \subset \mathbb{R}^{n}$ containing $v$. Then, for any integer $\rho \geq 0$ and any multi-index $\beta$ with $|\beta| \leq \rho$ :

$$
D^{\beta} \mathcal{T}_{v, \rho} f=\mathcal{T}_{v, \rho-|\beta|} D^{\beta} f .
$$

Proof From the definition of $\mathcal{T}_{v, \rho}$, for $x \in B$ :

$$
\begin{aligned}
D^{\beta} \mathcal{T}_{v, \rho} f(x) & =\sum_{\substack{|\alpha| \leq \rho \\
\alpha \geq \beta}} \frac{D^{\alpha} f(v)}{(\alpha-\beta) !}\left(x_{1}-v_{1}\right)^{\alpha_{1}-\beta_{1}} \cdots\left(x_{n}-v_{n}\right)^{\alpha_{n}-\beta_{n}} \\
& =\sum_{|\alpha| \leq \rho-|\beta|} \frac{D^{\alpha+\beta} f(v)}{\alpha !}\left(x_{1}-v_{1}\right)^{\alpha_{1}} \cdots\left(x_{n}-v_{n}\right)^{\alpha_{n}}=\mathcal{T}_{v, \rho-|\beta|} D^{\beta} f(x) .
\end{aligned}
$$


From Lemmas 1 and 2, we obtain:

Lemma 3 Let $v, w, e, B$ be as in Lemma 1. Suppose that $f, g \in C^{\infty}(B)$ and that for some $r \geq 0$ :

$$
\left.D^{\beta} f\right|_{e}=\left.D^{\beta} g\right|_{e}, \quad|\beta| \leq r .
$$

Then, for any $\rho \geq 0$ :

$$
\left.D^{\beta} \mathcal{T}_{v, \rho} f\right|_{e}=\left.D^{\beta} \mathcal{T}_{v, \rho} g\right|_{e}, \quad|\beta| \leq r .
$$

Proof If $|\beta|>\rho$, Eq. (2) trivially holds since both sides are equal to 0 . If $|\beta| \leq \rho$, by Lemma 2, Eq. (2) is equivalent to:

$$
\left.\mathcal{T}_{v, \rho-|\beta|} D^{\beta} f\right|_{e}=\left.\mathcal{T}_{v, \rho-|\beta|} D^{\beta} g\right|_{e},
$$

and by Lemma 1 , this is implied by equation (1).

\section{Characterization of supersmoothness}

We are now approaching a characterization of supersmoothness.

\subsection{Polynomial spline spaces}

For integers $r$ and $d$ with $0 \leq r \leq d$, let

$$
S_{d}^{r}(\Delta):=\left\{s \in C^{r}(\Omega):\left.s\right|_{T} \in \Pi_{d}, T \in \Delta\right\},
$$

where $\Pi_{d}$ is the linear space of polynomials in $\mathbb{R}^{n}$ of degree at most $d$. Thus, $S_{d}^{r}(\Delta)$ is the usual linear space of polynomial splines on $\Delta$ of smoothness $r$ and degree at most $d$.

\subsection{Degeneracy}

Consider an interior cell $\Delta_{v}$. By definition, for any $r \geq 0$, we have $\Pi_{d} \subset S_{d}^{r}\left(\Delta_{v}\right)$. Sometimes, however, depending on $\Delta_{v}$ and $r$, we might have $S_{d}^{r}\left(\Delta_{v}\right)=\Pi_{d}$. In this case, $S_{d}^{r}\left(\Delta_{v}\right)$ contains no "true" splines, only polynomials, and we view $S_{d}^{r}\left(\Delta_{v}\right)$ as being degenerate in this sense.

Definition 1 We will say that $S_{d}^{r}\left(\Delta_{v}\right)$ is degenerate if $S_{d}^{r}\left(\Delta_{v}\right)=\Pi_{d}$.

As an example, the space $S_{r}^{r}\left(\Delta_{v}\right)$ is degenerate for any $r \geq 0$.

\subsection{Piecewise Taylor approximations}

Next, recall the more general set of splines $S\left(\Delta_{v}\right)$ and let:

$$
\sigma=\left\{\left(U_{T}, f_{T}\right): T \in \Delta_{v}\right\} \in S\left(\Delta_{v}\right) .
$$


For any $\rho \geq 0$, we can make the following piecewise Taylor approximation of $\sigma$ :

$$
\mathcal{T}_{v, \rho} \sigma:=\left\{\left(\mathbb{R}^{n}, \mathcal{T}_{v, \rho} f_{T}\right): T \in \Delta_{v}\right\} \in S\left(\Delta_{v}\right)
$$

Due to Lemma 3, we next show:

Lemma 4 If $\sigma \in S^{r}\left(\Delta_{v}\right)$ for any $r \geq 0$ then $\mathcal{T}_{v, \rho} \sigma \in S^{r}\left(\Delta_{v}\right)$ for any $\rho \geq 0$.

Proof Let $T_{1}, T_{2} \in \Delta_{v}$ be two $n$-simplices that share a common $(n-1)$-face $F$. The face $F$ is the union of all the line segments $e$ that connect $v$ to the $(n-2)$-dimensional face of $F$ opposite to $v$. The pieces $f_{T_{1}}$ and $f_{T_{2}}$ have the same derivatives up to order $r$ on $e$. Therefore, by Lemma 3, the two Taylor approximations $\mathcal{T}_{v, \rho} f_{T_{1}}$ and $\mathcal{T}_{v, \rho} f_{T_{2}}$ have the same derivatives up to order $r$ on $e$. Therefore, they have the same derivatives up to order $r$ on the whole face $F$. Thus, $\mathcal{T}_{v, \rho} \sigma \in S^{r}\left(\Delta_{v}\right)$ as claimed.

\subsection{Characterization}

With the previous definitions in place, the characterization is as follows.

Theorem 1 Let $\Delta_{v}$ be an interior cell and suppose $0 \leq r \leq \rho$. Then, $S^{r}\left(\Delta_{v}\right) \subset$ $C^{\rho}\left(\Delta_{v}\right)$ if and only if $S_{\rho}^{r}\left(\Delta_{v}\right)$ is degenerate.

Proof Suppose that $S_{\rho}^{r}\left(\Delta_{v}\right)$ is degenerate and let:

$$
\sigma=\left\{\left(U_{T}, f_{T}\right): T \in \Delta_{v}\right\} \in S^{r}\left(\Delta_{v}\right) .
$$

By Lemma $4, \mathcal{T}_{v, \rho} \sigma \in S^{r}\left(\Delta_{v}\right)$. Therefore, we can define a polynomial spline $s \in$ $S_{\rho}^{r}\left(\Delta_{v}\right)$, by:

$$
\left.s\right|_{T}=\mathcal{T}_{v, \rho} f_{T}, \quad T \in \Delta_{v} .
$$

By the assumption that $S_{\rho}^{r}\left(\Delta_{v}\right)$ is degenerate, $s \in \Pi_{\rho}$. Then, for any $T_{1}, T_{2} \in \Delta_{v}$,

$$
D^{\alpha} f_{T_{1}}(v)=D^{\alpha} \mathcal{T}_{v, \rho} f_{T_{1}}(v)=D^{\alpha} s(v)=D^{\alpha} \mathcal{T}_{v, \rho} f_{T_{2}}(v)=D^{\alpha} f_{T_{2}}(v), \quad|\alpha| \leq \rho .
$$

This proves that $S^{r}\left(\Delta_{v}\right) \subset C^{\rho}\left(\Delta_{v}\right)$.

Conversely, suppose that $S^{r}\left(\Delta_{v}\right) \subset C^{\rho}\left(\Delta_{v}\right)$ and let $s \in S_{\rho}^{r}\left(\Delta_{v}\right)$. Then, we can define:

$$
\sigma=\left\{\left(\mathbb{R}^{n},\left.s\right|_{T}\right): T \in \Delta_{v}\right\} \in S^{r}\left(\Delta_{v}\right) .
$$

The assumption that $S^{r}\left(\Delta_{v}\right) \subset C^{\rho}\left(\Delta_{v}\right)$ implies that $\sigma \in C^{\rho}\left(\Delta_{v}\right)$. Therefore, for any $T_{1}, T_{2} \in \Delta_{v}$,

$$
\left.D^{\alpha} s\right|_{T_{1}}(v)=\left.D^{\alpha} s\right|_{T_{2}}(v), \quad|\alpha| \leq \rho .
$$

Since $\left.s\right|_{T_{1}},\left.s\right|_{T_{2}} \in \Pi_{\rho}$, this implies that $\left.s\right|_{T_{1}}=\left.s\right|_{T_{2}}$. Thus, $s \in \Pi_{\rho}$. This proves that $S_{\rho}^{r}\left(\Delta_{v}\right)$ is degenerate.

We remark that this theorem also holds if we reduce the smoothness assumption on the pieces $f_{T}$ of the splines $\sigma$ in $S^{r}\left(\Delta_{v}\right)$ to being in $C^{\rho}\left(U_{T}\right)$ instead of in $C^{\infty}\left(U_{T}\right)$. 


\subsection{Maximal order of supersmoothness}

We can also consider the mos (maximal order of supersmoothness) of $S^{r}\left(\Delta_{v}\right)$, i.e.,

$$
\operatorname{mos} S^{r}\left(\Delta_{v}\right):=\max \left\{\rho \geq r: S^{r}\left(\Delta_{v}\right) \subset C^{\rho}\left(\Delta_{v}\right)\right\} .
$$

To characterize this, observe that we have a nested sequence of spaces:

$$
\Pi_{r}=S_{r}^{r}\left(\Delta_{v}\right) \subset S_{r+1}^{r}\left(\Delta_{v}\right) \subset S_{r+2}^{r}\left(\Delta_{v}\right) \subset \cdots .
$$

Therefore, if $S_{d}^{r}\left(\Delta_{v}\right)$ is non-degenerate for some $d \geq r$, then $S_{k}^{r}\left(\Delta_{v}\right)$ is nondegenerate for all $k \geq d$. Thus, for any cell $\Delta_{v}$ and any $r \geq 0$, there is a unique highest degree $d \geq r$ such that $S_{d}^{r}\left(\Delta_{v}\right)$ is degenerate. From Theorem 1, we deduce:

Corollary $1 \operatorname{mos} S^{r}\left(\Delta_{v}\right)=\max \left\{d \geq r: S_{d}^{r}\left(\Delta_{v}\right)\right.$ is degenerate $\}$.

\section{Applications}

We now apply the characterization theorem to some concrete examples. For a cell $\Delta_{v}$ in $\mathbb{R}^{n}$ and smoothness $r \geq 0$ the spline space $S_{d}^{r}\left(\Delta_{v}\right)$, with $d \geq r$, is degenerate if:

$$
\operatorname{dim} S_{d}^{r}\left(\Delta_{v}\right)=\operatorname{dim} \Pi_{d}=\left(\begin{array}{c}
d+n \\
n
\end{array}\right) .
$$

For some cell configurations, degeneracy is known for specific degrees $d>r$. We then conclude from Theorem 1 that all splines in $S^{r}\left(\Delta_{v}\right)$ have supersmoothness of order $d$, but we do not know whether $d$ is optimal. However, if we know the dimensions of all the spaces $S_{d}^{r}\left(\Delta_{v}\right), d>r$, we obtain the maximal supersmoothness from Corollary 1 by finding the largest $d$ satisfying (3).

We note also that Alfeld [1] has computed the dimension of many spline spaces over various kinds of cell. These computational results also determine supersmoothness by Theorem 1 or Corollary 1 .

\subsection{Clough-Tocher split}

In $\mathbb{R}^{2}$, when $\Delta_{v}$ has three triangles, it is a Clough-Tocher split, $\Delta_{C T}$, and, using the theory of Bernstein-Bézier polynomials, Farin showed in [5, Theorem 7] that $S_{r+1}^{r}\left(\Delta_{C T}\right)$ is degenerate for any $r \geq 1$. He then concluded in [5, Corollary 8] that the pieces of any spline in $S_{d}^{r}\left(\Delta_{C T}\right), 1 \leq r \leq d$, have matching derivatives of order $r+1$ at $v$.

We can now apply Theorem 1 to conclude more generally that $S^{r}\left(\Delta_{C T}\right) \subset$ $C^{r+1}\left(\Delta_{C T}\right)$ for $r \geq 1$. However, this is not optimal supersmoothness for general $r$.

\subsection{An arbitrary cell in 2D}

Sorokina made a substantial generalization of Farin's result. She showed in [14, Theorem 3.1] that if $\Delta_{v}$ has $m$ triangles, and the $m$ interior edges have different slopes, 
then for $0 \leq r \leq d$, the pieces of any spline $s \in S_{d}^{r}\left(\Delta_{v}\right)$ have matching derivatives at $v$ up to order:

$$
\rho=r+\left\lfloor\frac{r+1}{m-1}\right\rfloor \text {. }
$$

The proof was based on comparing the dimension of $S_{d}^{r}(\Delta)$ with those of superspline spaces. Since $\rho$ in (4) is independent of the degree $d$, one might expect a more general result. This was also suggested by the work of Shekhtman and Sorokina [12]. From (4), it follows that there is at least one order of supersmoothness when $r \geq m-2$ for any degree $d \geq r$. Shekhtman and Sorokina showed that this is also true for more general splines, in other words, in our notation, $S^{r}\left(\Delta_{v}\right) \subset C^{r+1}\left(\Delta_{v}\right)$ when $r \geq$ $m-2$. Their proof was based on expressing partial derivatives as linear combinations of directional derivatives along the edges meeting at $v$. Using Corollary 1 , we can now improve this result to match that of the polynomial case. To do this, we first transform the dimension formula of Lai and Schumaker [8] into a more suitable form.

Lemma 5 Suppose $\Delta_{v}$ has $m$ triangles and suppose there are $m_{v}$ different slopes among the interior edges of $\Delta_{v}$. For $0 \leq r \leq d$ :

$$
\operatorname{dim} S_{d}^{r}\left(\Delta_{v}\right)=\operatorname{dim} \Pi_{d}+\left(m-m_{v}\right) \operatorname{dim} \Pi_{d-r-1}+\sum_{j=1}^{d-r}\left(\tau_{v, j}\right)_{+},
$$

where $\tau_{v, j}:=j\left(m_{v}-1\right)-(r+1)$, and $(x)_{+}:=x$ if $x>0$ and $(x)_{+}:=0$ otherwise.

Proof The dimension of $S_{d}^{r}\left(\Delta_{v}\right)$ was derived in [8, Theorem 9.3] in the form:

$$
\operatorname{dim} S_{d}^{r}\left(\Delta_{v}\right)=\left(\begin{array}{c}
r+2 \\
2
\end{array}\right)+m\left(\begin{array}{c}
d-r+1 \\
2
\end{array}\right)+\sum_{j=1}^{d-r}\left(-\tau_{v, j}\right)_{+}
$$

Using the fact that:

$$
\sum_{j=1}^{d-r} \tau_{v, j}=m_{v} \sum_{j=1}^{d-r} j-\sum_{j=1}^{d-r}(r+j+1)=m_{v}\left(\begin{array}{c}
d-r+1 \\
2
\end{array}\right)-\left(\begin{array}{c}
d+2 \\
2
\end{array}\right)+\left(\begin{array}{c}
r+2 \\
2
\end{array}\right),
$$

we can rewrite (6) as:

$$
\operatorname{dim} S_{d}^{r}\left(\Delta_{v}\right)=\operatorname{dim} \Pi_{d}+\left(m-m_{v}\right)\left(\begin{array}{c}
d-r+1 \\
2
\end{array}\right)+\sum_{j=1}^{d-r}\left(\left(-\tau_{v, j}\right)_{+}+\tau_{v, j}\right) .
$$

Then, using the fact that $(-x)_{+}+x=x_{+}$, the result follows.

Theorem 2 Suppose $\Delta_{v}$ has $m$ triangles and suppose there are $m_{v}$ different slopes among the interior edges of $\Delta_{v}$. Then, for $r \geq 0$ :

$$
\operatorname{mos} S^{r}\left(\Delta_{v}\right)= \begin{cases}r+\left\lfloor\frac{r+1}{m-1}\right\rfloor, & m_{v}=m ; \\ r, & m_{v}<m .\end{cases}
$$


Proof By Corollary 1, it is sufficient to determine the highest degree $d \geq r$ such that $S_{d}^{r}\left(\Delta_{v}\right)$ is degenerate, i.e., such that $\operatorname{dim} S_{d}^{r}\left(\Delta_{v}\right)=\operatorname{dim} \Pi_{d}$. To do this, we use Lemma 5. Suppose $m_{v}<m$. If $d=r+1$, the second term in (5) is strictly positive and so $S_{r+1}^{r}\left(\Delta_{v}\right)$ is non-degenerate. Therefore, $S_{d}^{r}\left(\Delta_{v}\right)$ is degenerate if and only if $d=r$. Otherwise, $m_{v}=m$. Then, considering the third term in (5), $S_{d}^{r}\left(\Delta_{v}\right)$ is degenerate if and only if $\tau_{v, j} \leq 0$ for all $j=1, \ldots, d-r$, or equivalently $\tau_{v, d-r} \leq 0$, which is equivalent to:

$$
d \leq r+\left\lfloor\frac{r+1}{m-1}\right\rfloor \text {. }
$$

As an example, for the Clough-Tocher split, we have $m=m_{v}=3$ and so

$$
\operatorname{mos} S^{r}\left(\Delta_{C T}\right)=r+\left\lfloor\frac{r+1}{2}\right\rfloor \text {. }
$$

\subsection{The Alfeld split in $\mathbb{R}^{n}$}

The dimensions of the spaces $S_{d}^{r}\left(\Delta_{v}\right)$ are not currently known for a general cell $\Delta_{v}$ in $\mathbb{R}^{n}$ for $n \geq 3$. However, they are known in special cases. One of these is the Alfeld split in $\mathbb{R}^{n}$. In $\mathbb{R}^{n}, n \geq 2$, the split is constructed by choosing any $n$-dimensional simplex $T$ and splitting it into $n+1$ smaller simplices by choosing an arbitrary interior point $v$ in $T$ and connecting it to each of the $n+1$ faces (of dimension $n-1$ ) of $T$. We denote this split by $\Delta_{A, n}$. The $3 \mathrm{D}$ case $\Delta_{A, 3}$ is shown in Fig. 2.

Using the theory of Bernstein-Bézier polynomials, Worsey and Farin showed in [16, Lemma 3.1] that $S_{2}^{1}\left(\Delta_{A, n}\right)$ is degenerate. From this, Theorem 1 implies that $S^{1}\left(\Delta_{A, n}\right) \subset C^{2}\left(\Delta_{A, n}\right)$. But we can make a further generalization by invoking the recently derived dimension formula of Foucart and Sorokina [6] and Schenck [9]. Let us define, for $n \geq 1$ and $r \geq 0$ :

$$
\rho_{n, r}:=r+(n-1)\left\lfloor\frac{r+1}{2}\right\rfloor .
$$

Theorem 3 The maximal order of supersmoothness of the Alfeld split is:

$$
\operatorname{mos} S^{r}\left(\Delta_{A, n}\right)=\rho_{n, r} .
$$

Proof The dimensions of the polynomial spline spaces on the Alfeld split were generated and conjectured by Foucart and Sorokina [6] and proved by Schenck [9]: for $0 \leq r \leq d$

$$
\operatorname{dim} S_{d}^{r}\left(\Delta_{A, n}\right)=\operatorname{dim} \Pi_{d}+A(n, d, r),
$$

where

$$
A(n, d, r)=\left\{\begin{array}{l}
n\left(\begin{array}{c}
d+n-(r+1)(n+1) / 2 \\
n \\
\left(\begin{array}{c}
d+n-1-r(n+1) / 2 \\
n
\end{array}\right)+\cdots+\left(\begin{array}{c}
d-r(n+1) / 2 \\
n
\end{array}\right),
\end{array} \quad \text { if } r\right. \text { is even. }
\end{array}\right.
$$

Therefore, $S_{d}^{r}\left(\Delta_{A, n}\right)$ is degenerate if and only if $A(n, d, r)=0$, or equivalently if

$$
\left\{\begin{array}{l}
d-(r+1)(n+1) / 2 \leq-1, \quad \text { if } r \text { is odd; } \\
d-1-r(n+1) / 2 \leq-1, \quad \text { if } r \text { is even. }
\end{array}\right.
$$


By Corollary 1, the maximal order of supersmoothness is the largest such $d$, i.e.,

$$
d=\left\{\begin{array}{l}
(r+1)(n+1) / 2-1, \quad \text { if } r \text { is odd; } \\
r(n+1) / 2, \quad \text { if } r \text { is even, }
\end{array}\right.
$$

or equivalently, $d=\rho_{n, r}$.

For example, $S^{1}\left(\Delta_{A, n}\right) \subset C^{n}\left(\Delta_{A, n}\right)$, and in particular, $S^{1}\left(\Delta_{A, 3}\right) \subset C^{3}\left(\Delta_{A, n}\right)$, which shows that the $C^{1}$ macro-element on the Alfeld split $\Delta_{A, 3}$ described in [8, Section 18.3] has supersmoothness of order 3.

We note that Theorem 3 in the case $n=2$ agrees with the supersmoothness of the Clough-Tocher split in equation (8).

\subsection{The $\Delta_{k, n}$ split}

Worsey and Farin [16] proposed an alternative generalization of the Clough-Tocher split to $\mathbb{R}^{n}$, using recursion through the Euclidean dimensions; see also [13]. To split an $n$-simplex $T$, they first split the faces of $T$ of dimension 2 (triangles) by making a Clough-Tocher split. They next split each 3-face (a tetrahedron) $F$ of $T$ by choosing any point in the relative interior of $F$ and connecting it to the twelve triangles on the boundary of $F$ constructed in the previous step. They continue in a similar way, next splitting faces of $T$ of dimension 4 and so on. Part of a Worsey-Farin split in 3D is shown in Fig. 3, viewed as a refinement of an Alfeld split. One of the subsimplices of the Alfeld split has been split into three.

Let us consider a more general split. We choose any Euclidean dimension $k, 1 \leq$ $k \leq n$. We then initialize the splitting by splitting each $k$-face $F$ of $T$ by choosing any point in the relative interior of $F$ and connecting it to the $(k-1)$-faces of $F$. Then, for $j=k+1, \ldots, n$ in sequence, we split each $j$-face $F$ of $T$ by choosing any point in the relative interior of $F$ and connecting it to the

$$
(j+1) \times \frac{j !}{k !}=\frac{(j+1) !}{k !}
$$

Fig. 3 Part of a Worsey-Farin split in 3D

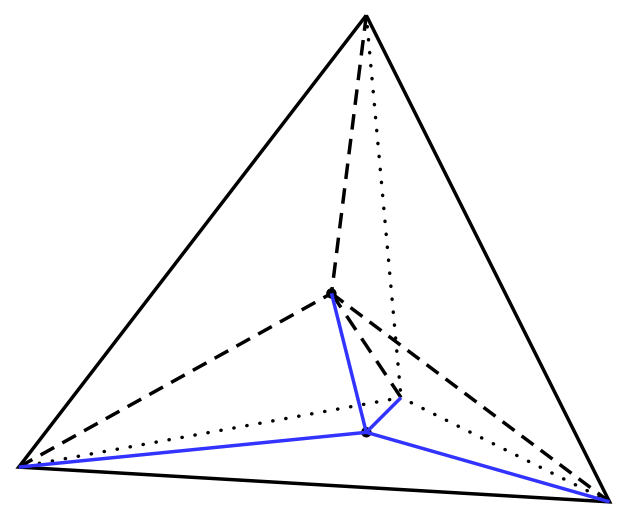


simplices of dimension $(j-1)$ on the boundary of $F$ constructed in the previous step. The resulting split of $T$ is a cell around the point $v$ in the interior of $T$ chosen at the last step $(j=n)$. It has $(n+1) ! / k$ ! sub-simplices and we denote it by $\Delta_{k, n}$.

For example, in $2 \mathrm{D}, \Delta_{2,2}$ is a Clough-Tocher split and $\Delta_{1,2}$ is a Powell-Sabin 6-split. In $3 \mathrm{D}, \Delta_{3,3}$ is an Alfeld split, $\Delta_{2,3}$ is a Worsey-Farin split and $\Delta_{1,3}$ is a Worsey-Piper split.

By construction, each of the $(n-1)$-faces of $T$ is itself split into a $\Delta_{k, n-1}$ split. A split $\Delta_{k, n}, k<n$, can also be viewed as a refinement of a split $\Delta_{k+1, n}$.

It was shown by Worsey and Farin [16] that $S_{2}^{1}\left(\Delta_{2, n}\right)$ is degenerate for any $n \geq 2$. Based on this observation, they concluded, as "an interesting aside," that their $C^{1}$ piecewise-cubic element has supersmoothness of order 2 at $v$. Theorem 1 implies more generally that $S^{1}\left(\Delta_{2, n}\right) \subset C^{2}\left(\Delta_{2, n}\right)$. Using now degeneracy over the Alfeld split in $\mathbb{R}^{k}$, we obtain a more general result.

Theorem 4 The maximal order of supersmoothness of a $\Delta_{k, n}$ split, $2 \leq k \leq n$, is bounded as follows:

$$
\rho_{k, r} \leq \operatorname{mos} S^{r}\left(\Delta_{k, n}\right) \leq \rho_{n, r} .
$$

Proof First let $r \leq d \leq \rho_{k, r}$. We will show that $S_{d}^{r}\left(\Delta_{k, n}\right)$ is degenerate. The proof of this is similar to that of [16, Theorem 3.2] and is by induction on $n \geq k$. Consider first $n=k$. Since $\Delta_{k, k}$ is a $k$-dimensional Alfeld split, it follows from Lemma 5 that $S_{d}^{r}\left(\Delta_{k, k}\right)$ is degenerate. Now suppose $n>k$ and let $s \in S_{d}^{r}\left(\Delta_{k, n}\right)$. Let $F$ be one of the $(n-1)$-faces of $T$. Let $w$ be the point in the relative interior of $F$ used to make the $(n-1)$-dimensional split $\Delta_{k, n-1}(F)$ of $F$ in the construction of $\Delta_{k, n}$. For each $\lambda \in(0,1]$, let $F_{\lambda}$ be the $(n-1)$-simplex:

$$
F_{\lambda}=\{(1-\lambda) v+\lambda x: x \in F\},
$$

which is parallel to $F$ and passes through the point:

$$
p=(1-\lambda) v+\lambda w .
$$

The split $\Delta_{k, n-1}(F)$ induces an analogous split $\Delta_{k, n-1}\left(F_{\lambda}\right)$. By the induction hypothesis, $S_{d}^{r}\left(\Delta_{k, n-1}\left(F_{\lambda}\right)\right)$ is degenerate and so all the pieces of $s$ meeting at $[v, w]$ have common derivatives within $F_{\lambda}$ up to order $d$ at $p$. Since all these pieces join continuously along $[v, w]$, they also have common derivatives along $[v, w]$. Therefore, all these pieces are the same polynomial and thus $s$ belongs to $S_{d}^{r}\left(\Delta_{A, n}\right)$. Since $d \leq \rho_{k, r} \leq \rho_{n, r}$, it follows, as in the proof of Theorem 3, that $s \in \Pi_{d}$.

This proves the lower bound on $\operatorname{mos} S^{r}\left(\Delta_{\mathrm{WF}, n}\right)$. To prove the upper bound, we just need to observe that $\Delta_{k, n}$ is a refinement of an Alfeld split $\Delta_{A, n}$, which implies that:

$$
S_{d}^{r}\left(\Delta_{A, n}\right) \subset S_{d}^{r}\left(\Delta_{k, n}\right)
$$

for any $0 \leq r \leq d$. Thus, if $S_{d}^{r}\left(\Delta_{A, n}\right)$ is non-degenerate, so is $S_{d}^{r}\left(\Delta_{k, n}\right)$.

\subsection{The $\Delta_{n-1, n}$ split}

Consider the special case of the $\Delta_{n-1, n}$ split, which has $n(n+1)$ subsimplices. It can be constructed by first making an Alfeld split $\Delta_{A, n}\left(=\Delta_{n, n}\right)$ of an $n$-simplex $T$ 
using some interior point $v$. We then choose an interior point of each boundary face $F$ (an $(n-1)$-simplex) of $T$ and use it to split $F$ into $n$ subsimplices and then connect them to $v$.

Let us say that $\Delta_{n-1, n}$ is aligned if, for every face $F$, the splitting point chosen for $F$ is the unique point in $F$ that is collinear with $v$ and the vertex of $T$ opposite $F$. This is what Schenck and Sorokina [10] called a facet split.

Theorem 5 The maximal order of supersmoothness of an aligned split $\Delta_{n-1, n}$ is

$$
\operatorname{mos} S^{r}\left(\Delta_{n-1, n}\right)=\rho_{n-1, r} .
$$

Proof The dimensions of the polynomial spline spaces for an aligned split $\Delta_{n-1, n}$ were derived by Schenck and Sorokina [10]. For $0 \leq r \leq d$ :

$$
\operatorname{dim} S_{d}^{r}\left(\Delta_{n-1, n}\right)=\operatorname{dim} \Pi_{d}+A(n, d, r)+(n+1) P(n, d, r),
$$

where $A(n, d, r)$ is as in Theorem 3 and

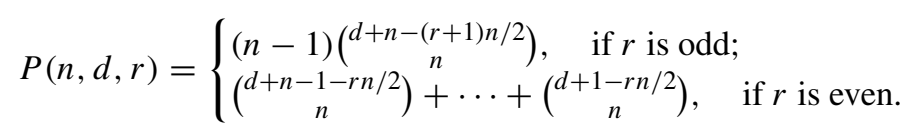

Therefore, $S_{d}^{r}\left(\Delta_{n-1, n}\right)$ is degenerate if and only if $A(n, d, r)+(n+1) P(n, d, r)=0$. Since $A(n, d, r)=0$ when $P(n, d, r)=0$, this is equivalent to the condition that $P(n, d, r)=0$, which holds when:

$$
\left\{\begin{array}{l}
d-(r+1) n / 2 \leq-1, \quad \text { if } r \text { is odd } \\
d-1-r n / 2 \leq-1, \quad \text { if } r \text { is even. }
\end{array}\right.
$$

The largest possible $d$ in both cases gives the result by Corollary 1 .

It is remarked in [10, Remark 4.3] that for $r=1$, the dimension formula (9) also holds even without the collinearity condition, from which we conclude that for an arbitrary $\Delta_{n-1, n}$ split:

$$
\operatorname{mos} S^{1}\left(\Delta_{n-1, n}\right)=\rho_{n-1,1}=n-1 .
$$

For example, in $3 \mathrm{D}$, for an arbitrary Worsey-Farin split $\Delta_{2,3}$ we have:

$$
\operatorname{mos} S^{1}\left(\Delta_{2,3}\right)=2 \text {. }
$$

\subsection{2-Cells}

Finally, we consider a slightly different kind of cell, constructed as follows. Let $T$ be an $n$-dimensional simplex and choose an interior point $v$ of $T$ and connect it to just one $(n-1)$-face of $T$, forming a simplex $T_{1}$ contained in $T$. We now let $T_{2}$ be the the closure of $T \backslash T_{1}$. The two elements $T_{1}$ and $T_{2}$ form what we will call a 2-cell, $\Delta_{2}=\left\{T_{1}, T_{2}\right\}$. Of course, it is not a cell of simplices because $T_{2}$ is not a simplex. Figure 4 shows a 2-cell in $2 D$.

Now, we can consider the supersmoothness of splines in $S^{r}\left(\Delta_{2}\right)$. Even though 2-cells do not occur in simplicial meshes, the local configuration of edges emanating from $v$ could occur in a polytopal mesh if we allowed non-convex polytopes. 
Fig. 4 A 2-cell in 2D

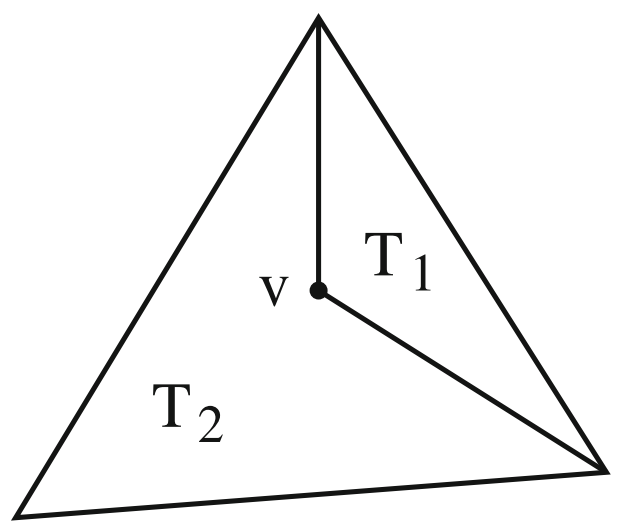

Shekhtman and Sorokina [12] studied this kind of configuration in 2D and showed that the order of supersmoothness is at least $r+1$ for any $r \geq 0$ (supersmoothness is "true" supersmoothness in this case, not just the matching of derivatives). We can now extend this result using our characterization. Even though a 2-cell contains the non-simplicial element $T_{2}$, the intersection of $T_{1}$ and $T_{2}$ is the union of $n$ faces (of dimension $(n-1))$ and so our characterization of supersmoothness at $v$ also holds for a 2-cell, i.e., we can apply Theorem 1 and Corollary 1 to a 2-cell $\Delta_{2}$. To use these results, we need the dimensions of the spline spaces $S_{d}^{r}\left(\Delta_{2}\right), 0 \leq r \leq d$.

Lemma 6 For any $0 \leq r \leq d$,

$$
\operatorname{dim} S_{d}^{r}\left(\Delta_{2}\right)=\operatorname{dim} \Pi_{d}+\operatorname{dim} \Pi_{d-n(r+1)} .
$$

Proof We have:

$$
\operatorname{dim} S_{d}^{r}\left(\Delta_{2}\right)=\operatorname{dim} \Pi_{d}+\operatorname{dim} S_{0}
$$

where

$$
S_{0}=\left\{s \in S_{d}^{r}\left(\Delta_{2}\right): s \equiv 0 \text { on } T_{2}\right\} .
$$

Letting $F_{1}, \ldots, F_{n}$ be the $(n-1)$-dimensional faces common to $T_{1}$ and $T_{2}$, we have:

$$
S_{0}=\left\{p \in \Pi_{d}:\left.D^{\alpha} p\right|_{F_{i}}=0,|\alpha| \leq r, i=1, \ldots, n\right\} .
$$

Let $l_{i}(x)=0$ be any equation for the face $F_{i}, i=1, \ldots, n$. Then, we can express any $p \in S_{0}$ uniquely in the form:

$$
p(x)=l_{1}(x)^{r+1} \cdots l_{n}(x)^{r+1} q(x), \quad x \in T_{1},
$$

where $q=0$ if $d-n(r+1)<0$ and $q \in \Pi_{d-n(r+1)}$ if $d-n(r+1) \geq 0$.

Theorem 6 For $r \geq 0, \operatorname{mos} S^{r}\left(\Delta_{2}\right)=r+(n-1)(r+1)$.

Proof By Lemma 6, $S_{d}^{r}\left(\Delta_{2}\right)$ is degenerate if and only if $\operatorname{dim} \Pi_{d-n(r+1)}=0$, or equivalently that $d-n(r+1)<0$. Thus, from Corollary 1 , the maximal order of supersmoothness is:

$$
d=n(r+1)-1=r+(n-1)(r+1) .
$$


For example, in $\mathbb{R}^{2}, \operatorname{mos} S^{r}\left(\Delta_{2}\right)=2 r+1$ and in $\mathbb{R}^{3}, \operatorname{mos} S^{r}\left(\Delta_{2}\right)=3 r+2$.

Funding Information Open Access funding provided by University of Oslo (incl Oslo University Hospital).

Open Access This article is licensed under a Creative Commons Attribution 4.0 International License, which permits use, sharing, adaptation, distribution and reproduction in any medium or format, as long as you give appropriate credit to the original author(s) and the source, provide a link to the Creative Commons licence, and indicate if changes were made. The images or other third party material in this article are included in the article's Creative Commons licence, unless indicated otherwise in a credit line to the material. If material is not included in the article's Creative Commons licence and your intended use is not permitted by statutory regulation or exceeds the permitted use, you will need to obtain permission directly from the copyright holder. To view a copy of this licence, visit http://creativecommonshorg/licenses/by/4.0/.

\section{References}

1. Alfeld, P.: MDS. http://www.math.utah.edu/alfeld/MDS/

2. Alfeld, P., Sorokina, T.: Linear differential operators on bivariate spline spaces and spline vector fields. BIT Numer. Math. 56, 15-32 (2016)

3. Arnold, D.N., Falk, R.S., Winther, R.: Finite element exterior calculus, homological techniques, and applications. Acta Numer. 15, 1-155 (2006)

4. Christiansen, S.H., Hu, K.: Generalized finite element systems for smooth differential forms and Stokes' problem. Numer. Math. 140, 327-371 (2018)

5. Farin, G.: Bézier polynomials over triangles and the construction of piecewise $C^{r}$ polynomials. Report TR/91. Uxbridge UK:Department of Mathematics, Brunel University, 1-66 (1980)

6. Foucart, S., Sorokina, T.: Generating dimension formulas for multivariate splines. Albanian J. Math. 7, 24-35 (2013)

7. Fu, G., Guzman, J., Neilan, M.: Exact smooth piecewise polynomial sequences on Alfeld splits. arXiv:1807.05883, 1-28 (2018)

8. Lai, M.-J., Schumaker, L.L.: Spline Functions on Triangulations, vol. 110. Cambridge University Press, Cambridge (2007)

9. Schenck, H.: Splines on the Alfeld split of a simplex and type a root systems. J Approx Theory 182, 1-6 (2014)

10. Schenck, H., Sorokina, T.: Subdivision and spline spaces. Constr. Approx. 47, 237-247 (2018)

11. Schumaker, L.L.: On super splines and finite elements. SIAM J. Numer. Anal. 26, 997-1005 (1989)

12. Shekhtman, B., Sorokina, T.: Intrinsic supersmoothness. J. Concr. Appl. Math. 13, 232-241 (2015)

13. Sorokina, T.: A $c^{1}$ multivariate clough-tocher interpolant. Constr. Approx. 29, 41-59 (2009)

14. Sorokina, T.: Intrinsic supersmoothness of multivariate splines. Numer. Math. 116, $421-434$ (2010)

15. Sorokina, T.: Redundancy of smoothness conditions and supersmoothness of bivariate splines. IMA J. Numer. Anal. 34, 1701-1714 (2014)

16. Worsey, A.J., Farin, G.: An n-dimensional clough-tocher interpolant. Constr. Approx. 3, 99-110 (1987)

Publisher's note Springer Nature remains neutral with regard to jurisdictional claims in published maps and institutional affiliations. 